\author{
Research Article
}

Qian Gao and Pingchuan Zhang*

\title{
The Errors Recognition and Compensation for the Numerical Control Machine Tools Based on Laser Testing Technology
}

https://doi.org/10.1515/phys-2019-0090

Received Nov 04, 2019; accepted Dec 02, 2019

\begin{abstract}
To improve the accuracy of numerical control machine tools error, this paper studies the application of laser testing technology in numerical control machine tool, and put forward a laser interferometer automatic aiming system which can conduct space error measurement. The system, in the process of the $\mathrm{NC}$ machine tool operating, can measure the machine tool space curve through changing the direction of the laser. The principle of laser interferometer and matlab analysis are adopted, through the experiment of laser interference technology in the numerical control machine tool, the law curve of error in $\mathrm{NC}$ machine tools is obtained, getting the method of error of NC machine tools. When conducting laser testing error experiment in NC machine tool, the results show that the laser testing can measure aggregated error and thermal error of $\mathrm{NC}$ machine tool better, with small control of environment and high accuracy of measurement.
\end{abstract}

Keywords: Numerical control machine tool; Laser detection technology; Error identification; compensation

PACS: 42.55.-f, 07.60.Ly

\section{Introduction}

The current NC machine tools develop very rapidly, and the accuracy of NC machining also increases significantly. But the positioning error of NC machine tools will also affected by scale error and geometric error, impacting on products processed by NC machining. In order to make

*Corresponding Author: Pingchuan Zhang: Pingchuan Zhang: School of Information Engineering, Henan institute of Science and Technology, Xinxiang 453003, China;

Email: zhangpingc@hist.edu.cn

Qian Gao: Department of Electrical and Electronic Engineering, Luohe Vocational Technology College, Luohe 462002, China
NC workshop produce high quality products, and also improve the productivity, it is urgent that realizing efficient and fast control on the error of machine tool in the production of NC machine tool, so we can get products with small error and high precision, achieving no error production. This has relatively high requirements on instruments and technical that measuring error.

The dual-frequency laser interferometer is high precise equipment measuring NC machine tools in contemporary. In the actual operation, the measuring technology of laser interference can accurately measure the actual position and ideal location in the process of operation, achieving the error measurement and the function of error compensation [1-4].

Professor Yang Jianguo, through the cooperation of foreign NC enterprises, has found a method using body diagonals to measure spatial position error in the process of NC machine tool operation, which is based on laser Doppler technique and multi-step measurement, error control in NC machine tool operation can be realized by using a simple laser, creating conditions for error compensation [5].

Mark A. V. Chapman has analyzed the measurement precision of laser vector diagonal from the principle of $\mathrm{NC}$ machine tool error forming, and measured the size of error accurately, also pointed out that the test data by using this method for error compensation may have some deficiencies.

H. Schwenke and others have proposed the laser tracking method based on the measurement of single laser tracking interferometer, this method cannot be influenced by external environment, namely, there has no direct relation with the run length of the NC machine tool, and the reference object is static, and this method can reduce the inaccuracy of measurement [6].

A team from Shanghai Jiaotong University, through the analysis of three-axis NC machine tool error, has proposed a measuring method of three-axis NC machine tool based on the raster, through detecting the linear motion trajectory precision in the three coordinate planes, the lin- 
earity error has been obtained, then through the three coordinate plane, the trajectory accuracy of circular motion is detected. The aggregate error of NC machine has been got effective control [7].

In the actual production of the NC machine tool, due to the limitation of machine itself and the work environment, $\mathrm{NC}$ machine tool will produce positioning error and straightness error, etc., influencing the products processed, especially for precision processing parts, small error will affect the precious parts, the automatic aiming test system studied can obtain the error in the process of operation, implementing the compensation of NC machine tool [8].

Therefore, this paper puts forward the error identification and compensation of CNC machine tools based on laser detection technology.

\section{Material and Methods}

As for all parts composing NC machine tool, they will produce error inevitable in the operation, and that each part will also produce error. Through analyzing the aspects of error generation in the processing, it has been found that when the machine tools in manufacturing the coordination of various assemblies will produce geometric error. Due to the high speed and high temperature, and if cooling is not timely, produce thermal error will be produced; the weight of NC machine tool is much heavier, in the process of cutting, it will produce vibration, and also cause the deformation in cutting; Servo system of machine tool, in the process of producing power, will also under the influence of voltage and current, causing errors; In addition. $\mathrm{NC}$ machine will also be affected by external factors. In the previous production, usually the direct measurement error is adopted, and the positioning error is taken as an example to show its principle [9-12].

\subsection{The direct measurement of positioning error in machine tool space}

Because the existing methods cannot directly measure error vector, the traditional method is a one-dimensional ball array measuring the error of single unit in NC machine tool, the analysis conducted also needs subsequent calculation and other studies, and the work needed to be done is very large. As shown in Figure 1, the one-dimensional ball sequence is neatly ranked above the machine tool, and then the position of one-dimensional sequence is ad- justed parallel to the position of the $\mathrm{X}$ axis. Through the three-dimensional camera on the principal axis of machine tool [13], the coordinates location of centre of each ball in ball series can be measured, the positioning of machine tools is the difference value, the formula used is shown as follows:

$$
\delta_{x}\left(X_{i}\right)=X_{i}-L_{i}
$$

Of which, $X_{i}$ refers to the center of ball distance value in the machine tool, $L_{i}$ refers to the reference value of distance between the balls, $\delta_{x}\left(X_{i}\right)$ refers to the positioning error values of $X$ coordinate axis in the location of $i$.



Figure 1: The structure diagram of one-dimensional ball measuring method

\subsection{The measurement principle of laser testing}

The measuring principle of laser interferometer is as shown in Figure 2. The principle of laser interferometer is achieved using the interference principle of light principle, and also combining with the Doppler Effect [14-16]. The two laser beams in Figure 2 have equal amplitude and different frequency, the specific working process is as follows: the laser 1 located in right sends out the light, and goes through $\lambda / 4$ slice in Figure 2, then the light changes into different linear polarized light. Using the optical splitter 3 , part of the light is reflected, therefore through analyzer 4 , the light will form different frequency signals, and then

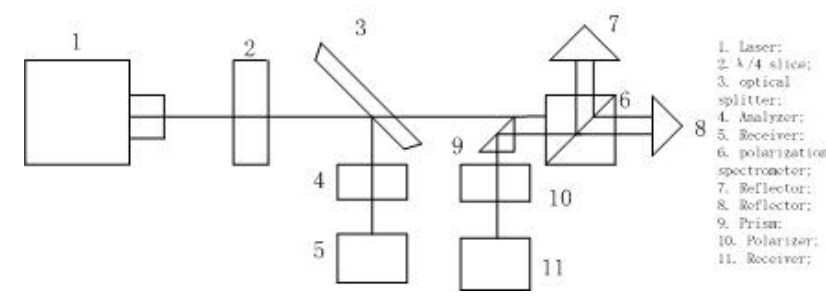

Figure 2: The measuring principle diagram of the laser interference 


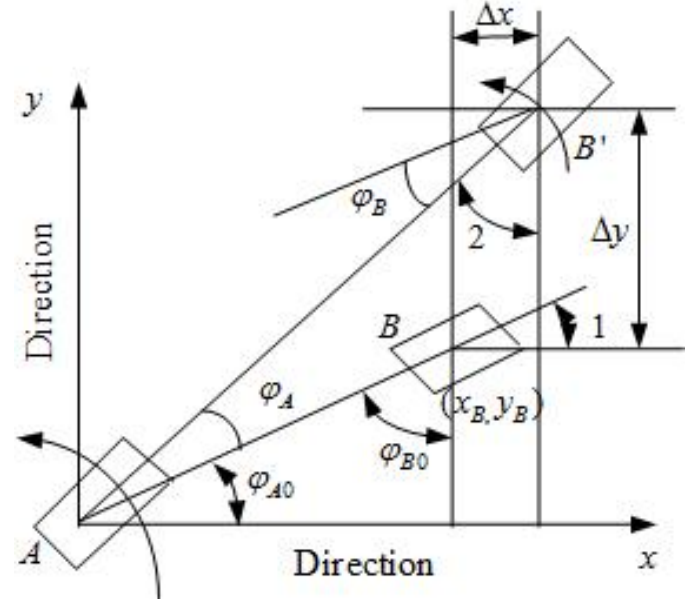

Figure 3: The reflection principle of xy plane

the signals are accepted by the receiver 5; This part of signal is known as the reference signal $[17,18]$. In the figure, the number 3 represents the optical splitter, and the rest of beam can be spread to polarization spectrometer 6 through the beam splitter, so the two beams, through their respective reflector 7 and 8 , finally converge to the polarization spectrometer. Through the reflection of prism 9, transfer to the polarizer 10 , finally received by receiver, this is the final measuring signal.

On the basis of the above laser interference, the alignment settings is increased, as for the working principle, it is as follows: the alignment principle of xy plane is as shown in Figure 3. A stands for interferoscope, B represents reflector, and $\mathrm{B}$ can be expressed as $\left(x_{B}, y_{B}\right)$, when the reflector $\mathrm{B}$ rotating to $\mathrm{B}$, the values in the direction $\mathrm{x}$, $\mathrm{y}$ increases $\Delta x$ and $\Delta y$, when rotating $\mathrm{B}$, the light beam occurs deflection, and the interferoscope should go around $\mathrm{z}$ rotating a certain angle to keep the position of the beam, the reflector should rotate to keep balance. In the figure, the rotating angle of interferoscope $\mathrm{A}$ at the beginning is as follows:

$$
\varphi_{A 0}=\arctan \left(y_{B} / x_{B}\right)
$$

The initial angle of the reflector B is as follows:

$$
\varphi_{B 0}=\arctan \left(x_{B} / y_{B}\right)
$$

The interferoscope should rotate the following angle:

$$
\begin{aligned}
\varphi_{A} & =\arctan \left[\left(y_{B}+\Delta y\right) /\left(x_{B}+\Delta x\right)\right] \\
& -\arctan \left(y_{B}+x_{B}\right)
\end{aligned}
$$

It can be seen from the figure that:

$$
\varphi_{B}=\angle 1-\angle 2=\varphi_{B 0}-\left[90-\left(\varphi_{A 0}+\varphi_{A}\right)\right]
$$

$$
=90-\varphi_{A 0}-90+\varphi_{A 0}+\varphi_{A}=\varphi_{A}
$$

From the above formula, it can be concluded that the angle of reflector is equal to that of interferoscope. The angle that the reflector should rotate can be obtained as follows:

$$
\varphi_{B}=\arctan \left[\left(y_{B}+\Delta y\right) /\left(x_{B}+\Delta x\right)\right]-\arctan \left(y_{B} / x_{B}\right)
$$

\section{Results}

\subsection{The error measurement of NC machine tool}

The experiment, adopting model XL-80 laser interferometer system, carries out direct measurement and also analysis on positioning error in coordinate axis and the error in the vertical direction of 3-axis NC machine tool. Due to the limited experimental conditions, the other errors are not analyzed. The positioning error is the difference between the actual position and target position, and it is an important index of machine tool accuracy, reflecting comprehensively the manufacturing assembly accuracy of mechanical part, feedback control accuracy of numerical control system and the thermal and mechanical coupling properties between the machine tool and environment.

The positioning error in $\mathrm{X}$ axis is as shown in Figure 4, the positioning error turns a downward trend as a whole, in the process of falling, the fluctuation is much smaller, and the overall trend is relatively stable, the maximum error is $15.1 \mu \mathrm{m}$, occurring in the location $\mathrm{x}=600 \mathrm{~mm}$.

As shown in Figure 5, compared with the positioning error in $\mathrm{X}$ axis, the change trend of the positioning error in $\mathrm{Y}$ axis presents the overall upward trend, though in the



Figure 4: The positioning error in $\mathrm{x}$ axis 




Figure 5: The positioning error in y axis



Figure 6: The error in vertical direction of $x$ axis

middle there is a relatively small fluctuations, the overall increasing state is relatively stable. Observing the error values, it can be found that the obtained value is relatively high, the maximum error is about $28.56 \mu \mathrm{m}$, lying in the location $\mathrm{y}=445 \mathrm{~mm}$ approximately. Because the gap existing between ball screws causes the error, so the error value will turn a rising trend with the increasing of y value.

Unlike the above positioning error, the straightness error in $\mathrm{X}$ axis shows very small. As shown in Figure 6, the straightness error in vertical direction of $\mathrm{X}$ axis shows a trend of increase first and then decrease during the route $0-130 \mathrm{~mm}$, afterward it increase dramatically during the route $130-280 \mathrm{~mm}$, it can be seen that the image start to fluctuate in the location $1.8 \mu \mathrm{m}$, and in the location about $x=400 \mathrm{~mm}$, the error produced by slide way in the location $400-600 \mathrm{~mm}$ is the largest, because products are often processed on the slide way, generating larger error. In general, it can be seen that if the value of $x$ is larger, the error generated shows a rising trend.

In the pitching angle error of $\mathrm{NC}$ machine tool, as for the $\mathrm{X}$ axis, the pitching angle error in the process of route increasing presents a downward trend, due to the error has nothing to do with the positive and negative, it can be seen that the error becomes larger with the increase of route, and the maximum lies in the location about $-56 \mathrm{um}$. It is as shown in Figure 7:

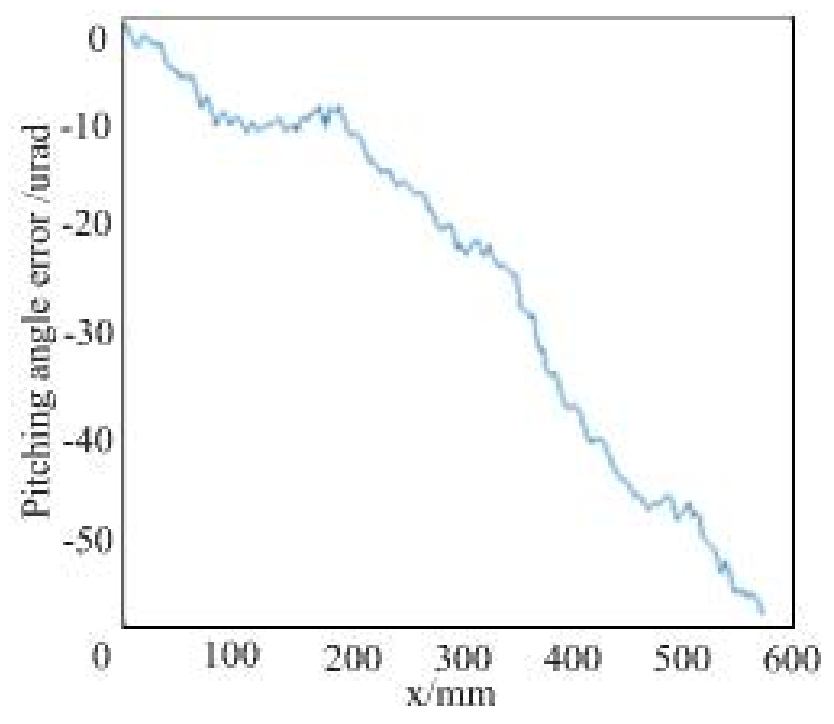

Figure 7: The pitching angle error in $\mathrm{x}$ axis

In the process of y axis movement, as shown in Figure 8 , the pitching angle error becomes larger with the increase of route $y$, the maximum is about $12 \mathrm{um}$, far less than the error in $\mathrm{X}$ axis, what's more it fluctuate more noticeable in the middle.

\subsection{The error compensation of NC machine tool}

Though the error of NC machine tool caused by many reasons, through a lot of experiments it can be seen that the error has its regularity, and the value of error will maintain relative stability in the position of machine tool movement. Therefore the error can be simple measured, getting the rule and obtaining the compensated values. As for the error of NC machine tool, the most fundamental thing is to realize the error detection in the process of machine tool movement, get the size of the deviation and find the rule, then conduct effective compensation of NC machine tools. 


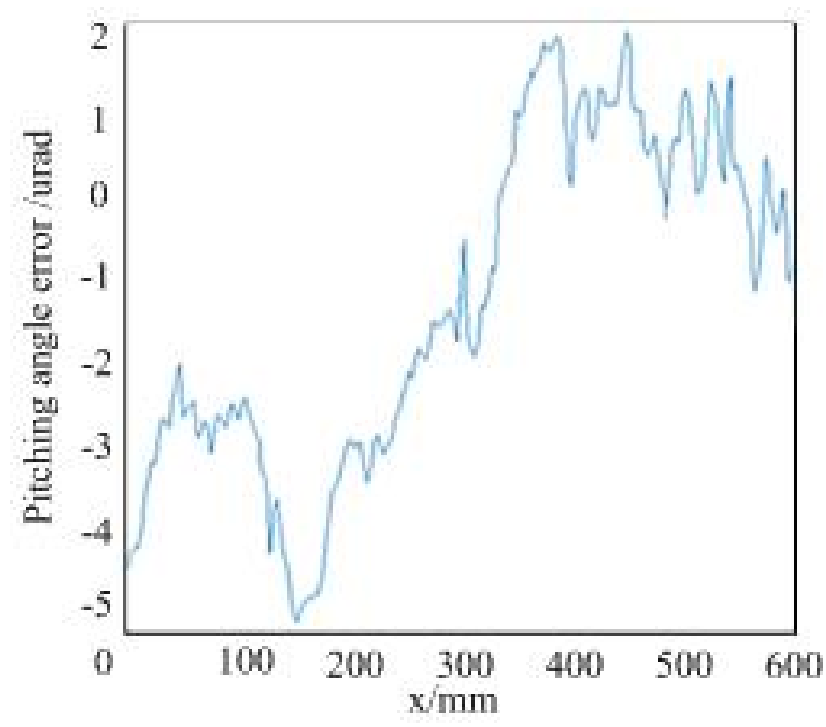

Figure 8: The pitching angle error in y axis

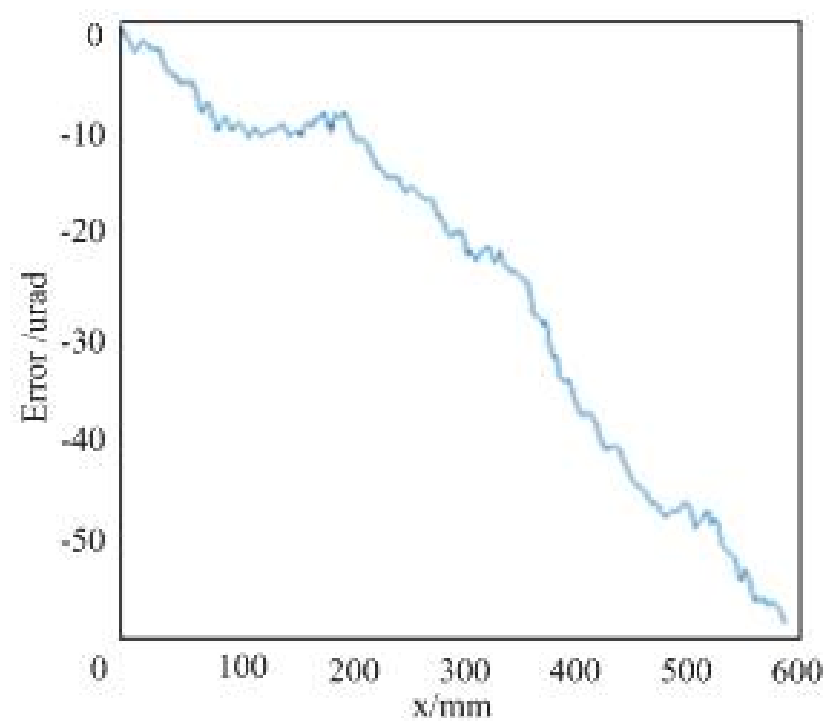

Figure 9: The error before compensation in $\mathrm{x}$ axis

The laser measurement technology not only measures the error of NC machine tool with high precision but also its automatic aiming system can realize error compensation.

The effect of compensation can be proved through experiment, and Figure 9 shows that before the compensation, the error range of laser interferometer measurement is between -15 and 10, it also can be seen that its error becomes larger with the increase of the route, the change trend is very obvious. In Figure 10, after the compensation of laser interferometer measurement error, it can be seen that the error range is between -4 and -1 , although the fluctuation in the middle is more noticeable, the amplitude

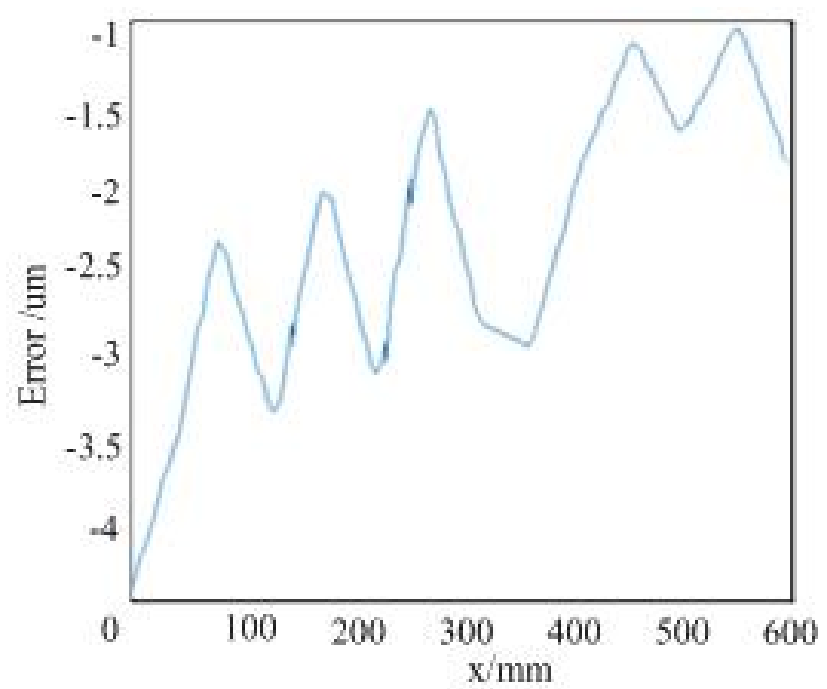

Figure 10: The error after ompensation in $\mathrm{x}$ axis

value is very small, realizing the processing compensation of NC machine tools. The error effect is very obvious.

In the current error detection of NC machine tool, thermal error is also existed. The forming reason of thermal error of NC machine tool is complex temperature field. In the process of machine tool movement the generated lots of heat and deformation make the machine tool produce thermal error. Measuring the temperature of machine tool at different time can get the changing rule of thermal error of machine tool, so the error amount can be obtained. In the aspect of thermal error, the sensor is installed to get the wanted value. The laser testing technology can measure the thermal error, and mathematical model is also needed to establish.

\section{Conclusion}

Laser testing technology, as the method measuring the machine tool error, has a good effect. The principle of automatic aiming of laser testing in the NC machine tool is studied, and the feasibility of automatic aiming system is proved. Based on the technology of laser testing, the error in the operation of the machine tool can be got, obtaining the deviation of NC machine tool more simple and accurate, and also conducting compensation effectively. The laser testing technology, as the high accuracy measuring technology, gets rid of traditional direct measurement shortcomings and improves the error precision of the NC machine tools. It has larger significance for the production of NC machine tools. But good feedback design isn’t 
achieved in this study, worthy to be studied continuous in the future.

Acknowledgement: This research was supported by Ministry of education: Beijing Bai Ke Rong Chuang Productionstudy Cooperation and Collaborative Education Project (No. 20160211006); Science and Technology Project of Henan Province Science and Technology Department (182102110301); Henan Education Department Project (18A210014).

\section{References}

[1] Peng W., Li D., Zhang M., et al., Characteristics of antibacterial molecular activities in poplar wood extractives, Saudi Journal of Biological Sciences, 2017, 24(2), 399-404.

[2] Yang Y., Zhong M., et al., Postolache, Internet of things for smart ports: Technologies and challenges, leee Instrumentation \& Measurement Magazine, 2018, 21(1), 34-43.

[3] Aladadi W.M., Moustafa M.F., et al., Genetic variability among seven cultivars of date palm (\&ITPhoenix dactylifera \&ITL.) based on embryonic DNA of old fruit, Kuwait Journal of Science, 2018, 45(1), 108-114.

[4] Kalaivani N., El-Maghrabi A.I., et al., Operation-compact spaces, regular spaces and normal spaces with -open sets in topological spaces, Journal of Interdisciplinary Mathematics, 2017, 20(2), 427-441.

[5] Ben Taher R., Naassi N., et al., On the Leslie matrices, Fibonacci sequences and population dynamics, Journal of Discrete Mathematical Sciences and Cryptography, 2017, 20(2), 565-594.

[6] Bonanno G., D’Agui G., et al., One-dimensional nonlinear boundary value problems with variable exponent, Discrete and Continuous Dynamical Systems-Series S, 2018, 11(2SI), 179-191.

[7] Iannizzotto A., Papageorgiou N.S., Existence and multiplicity results for resonant fractional boundary value problems, Discrete and Continuous Dynamical Systems-Series S, 2018, 11(3SI), 511532.
[8] Li R., Taylor M.M., The first zagreb index and some hamiltonian properties of the line graph of a graph, Journal of Discrete Mathematical Sciences and Cryptography, 2017, 20(2), 445-451.

[9] Hou J., Wen Z., Lai J., A constrained optimization reformulation of the generalized Nash equilibrium problem, Journal of Interdisciplinary Mathematics, 2017, 20(1), 27-34.

[10] Adiyaman M.E., Oger V., A residual method using Bezier curves for singular nonlinear equations of Lane-Emden type, Kuwait Journal of Science, 2017, 44(4), 9-18.

[11] Yang A., Han Y., Li S., Xing H., Pan Y., Liu W., Synthesis and comparison of photocatalytic properties for Bi2W06 nanofibers and hierarchical microspheres, Journal of Alloys and Compounds, 2017, 695, 915-921.

[12] Shen Y., Zhao N., Xia M., Du X., A deep q-learning network for ship stowage planning problem, Polish Maritime Research, 2017, 24(SI), 102-109.

[13] Gao W., Wang W.F., The fifth geometric-arithmetic index of bridge graph and carbon nanocones, Journal of Difference Equations and Applications, 2017, 23, 100-109.

[14] Malviya P.S., Yadav N., Ghosh S., Acousto-Optic modulation in ion implanted semiconductor plasmas having SDDC, Applied Mathematics \& Nonlinear Sciences, 2018, 3, 303-310.

[15] Mi C., Shen Y., Mi W.J., Huang Y.F., Ship identification algorithm based on 3D point cloud for automated ship loaders, Journal of Coastal Research, 2015, (73), 28-34.

[16] Nizami A.R., Perveen A., Nazeer W., Baqir M., WALK POLYNOMIAL: a new graph invariant, Applied Mathematics \& Nonlinear Sciences, 2018, 3, 321-330.

[17] Pandey P.K., Jaboob S.S.A., A finite difference method for a numerical solution of elliptic boundary value problems, Applied Mathematics \& Nonlinear Sciences, 2018, 3, 311-320.

[18] Vajravelu K., Li R., Dewasurendra M., Benarroch J., Ossi N., Zhang Y., Sammarco M., Prasad K.V., Effects of second-order slip and drag reduction in boundary layer flows, Applied Mathematics \& Nonlinear Sciences, 2018, 3, 291-302. 